\title{
DÉTERMINATION DES DÉPLACEMENTS THERMIQUES DES ATOMES DE SURFACE D'UN MONOCRISTAL DE CUIVRE (FACE 100) PAR L'ÉTUDE DE LA DIFFUSION ÉLASTIQUE D'UN JET ATOMIQUE D'HÉLIUM
}

\author{
G. ARMAND, J. LAPUJOULADE et Y. LEJAY \\ Service de Physique Atomique, Section d'Etudes des Interactions Gaz-Solides, \\ CEN Saclay, B.P. no 2, 91190 Gif-sur-Yvette, France
}

(Reçu le 18 mars 1976, accepté le 14 mai 1976)

\begin{abstract}
Résumé. - En utilisant un appareillage expérimental à résolution élevée on a pu séparer la partie élastique et cohérente de la réflexion spéculaire de l'hélium sur la face (100) du cuivre. Les variations de l'intensité de ce pic en fonction de la température du cristal et de l'énergie des atomes incidents s'interprètent suivant le modèle de Beeby à l'aide d'un facteur de Debye-Waller. On en tire la profondeur du puits de potentiel $\mathrm{He}-\mathrm{Cu}(0,009 \mathrm{eV})$ et une température de Debye de surface apparente $\left(\Theta_{\text {app }}=370 \mathrm{~K}\right)$. Un modèle simple de collision dans lequel l'hélium est en interaction avec quatre atomes de surface permet d'interpréter ce dernier résultat.
\end{abstract}

Abstract. - Using a high resolution experimental apparatus, the elastic and coherent part of the specular reflexion of helium on (100) copper has been isolated. The intensity dependence of this peak as a function of crystal temperatures or incident atom energy is rationalized, within the frame of Beeby's model, by means of a Debye-Waller factor. One obtains the $\mathrm{He}-\mathrm{Cu}$ potential well depth $(0.009 \mathrm{eV})$ and an apparent surface Debye temperature $\left(\Theta_{\text {app }}=370 \mathrm{~K}\right)$. A simple collision model when the helium atom is in interaction with four surface atoms may explain this last result.

Les atomes de surface d'un cristal ayant moins de voisins que ceux du cœur, on doit s'attendre à ce que leurs déplacements quadratiques moyens soient plus importants que ceux des atomes du volume ou, pour parler un autre langage, à ce que la température de Debye de surface $\Theta_{\mathrm{S}}$ soit inférieure à celle du volume $\Theta_{\mathrm{v}}$. Ces quantités étant reliées par la relation classique :

$$
\left\langle u^{2}\right\rangle=\frac{3 \hbar^{2} T_{\mathrm{S}}}{m_{\mathrm{s}} k \Theta^{2}}
$$

$T_{\mathrm{S}}:$ température du cristal,

$m_{\mathrm{s}}$ : masse des atomes du cristal.

Par exemple, pour les déplacements normaux des atomes d'une face (100) du Cuivre, Masri et Dobrynski ont calculé [1] $\Theta_{S_{\perp}}=240 \mathrm{~K}$ alors que pour le volume on $\mathrm{a} \Theta_{\mathrm{v}}=330 \mathrm{~K}$.

Cette valeur théorique peut être confrontée aux résultats obtenus en étudiant la dépendance thermique de l'intensité des pics de diffraction d'électrons lents (facteur de Debye-Waller). Les mesures de R. J. Reid [2] conduisent à $195 \mathrm{~K}<\Theta_{\mathrm{S}_{\perp}}<300 \mathrm{~K}$ selon l'énergie et l'incidence des électrons. Cette dispersion est due, d'une part, à la pénétration non négligeable des électrons dans le cristal et, d'autre part, aux effets dynamiques qui sont négligés dans l'interprétation cinématique utilisée par Reid et dont il est difficile de tenir compte.

En utilisant un faisceau d'atomes neutres comme sonde on peut espérer s'affranchir de ces difficultés. L'hélium qui se réfléchit sur les monocristaux propres de manière quasi élastique [3] se prête tout particulièrement à cette détermination. Les premières tentatives d'interprétation [4] n'ont pas, toutefois, été très convaincantes. En effet l'analyse de la dépendance thermique de l'intensité du pic de réflexion spéculaire de l'hélium à l'aide du facteur de Debye-Waller suppose que la réflexion est complètement élastique et cohérente. Une telle hypothèse peut paraître hasardeuse si l'on considère que :

1) Nous avons mis en évidence dans un précédent travail [5] que, dans le pic spéculaire, une fraction non négligeable des atomes avait été réflhéchie inélastiquement.

2) La cohérence de la réflexion a été très sérieusement mise en doute par G. Comsa [6] en raison, d'une part, de l'absence de pic de diffraction d'ordre supérieur à 00 et, d'autre part, de l'augmentation de 
l'intensité du pic spéculaire avec l'énergie incidente constatée par Merrill et al. [7] en flagrante contradiction avec les prédictions du facteur de DebyeWaller.

Nous avons essayé de préciser ces points en étudiant la réflexion de l'hélium sur une face (100) de Cuivre avec une résolution angulaire et énergétique nettement améliorée par rapport aux travaux antérieurs [15]. Le jet supersonique d'hélium est modulé par un hacheur mécanique et, après avoir diffusé sur le cristal, il parcourt une distance de vol de $50 \mathrm{~cm}$ avant d'être reçu sur un détecteur à ionisation. L'analyse numérique des spectres de temps de vol permet d'obtenir le flux réfléchi et, par déconvolution au moyen d'une transformée de Fourier discrète, la distribution de vitesse. L'énergie moyenne du jet incident peut être ajustée entre 0,063 et $0,27 \mathrm{eV}$ par chauffage thermique du gaz avant la tuyère [8]. La largeur relative de la distribution de vitesse est de l'ordre de $5 \%$. L'ouverture angulaire du jet est de $0,25^{\circ}$. L'ouverture du détecteur vue de la tuyère est également de $0,25^{\circ}$. Ceci représente un gain d'un facteur huit en résolution spatiale par rapport au dispositif antérieur [5]. Compte tenu de cette ouverture et de la dimension de l'échantillon $(20 \mathrm{~mm})$ on peut régler l'incidence entre 10 et $60^{\circ}$. L'échantillon est nettoyé, in situ, par bombardement ionique, la propreté est contrôlée par spectrométrie Auger. La température du cristal peut être régulée entre 373 et $1023 \mathrm{~K}$. Le porte-échantillon permet d'escamoter celui-ci afin de permettre, à tout moment, la mesure des caractéristiques du jet incident qui servent ainsi de référence.

Le résultat le plus marquant est que, pour les basses énergies incidentes, le lobe spatial de flux et la distribution de vitesse sont strictement identiques, aux erreurs expérimentales près, avant et après la diffusion. Une telle comparaison est faite sur les figures $1 a$ et $1 b$ pour une énergie incidente $E_{\mathrm{G}}=0,063 \mathrm{eV}$, un angle d'incidence $\theta_{\mathrm{i}}=60^{\circ}$ et une température de cristal $T_{\mathrm{S}}=373 \mathrm{~K}$. La partie inélastique détectée dans une expérience précédente [5] s'étendait environ sur cinq degrés de part et d'autre de la direction spéculaire aussi ne fournit-elle plus qu'une contribution

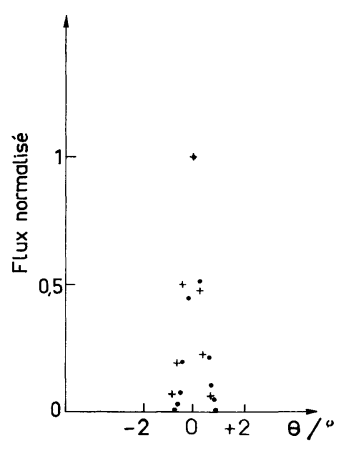

a)

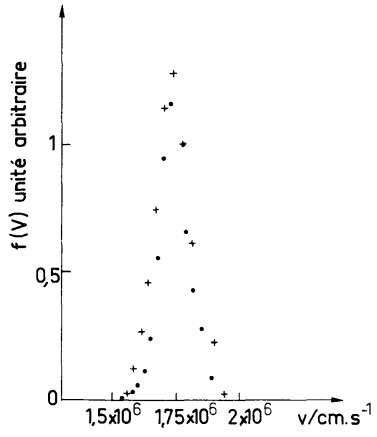

b)
FIG. 1. - $-a$ ) Lobes de flux (lobe réfléchi rapporté à la direction spéculaire). b) Distribution de vitesses dans la direction spéculaire. - faisceau incident, + faisceau diffusé. négligeable au flux entrant dans le détecteur à faible ouverture utilisé ici. Lorsque l'énergie incidente augmente l'intensité du pic élastique diminue fortement et pour les incidences voisines de la normale, on constate que la contribution inélastique devient significative. Les résultats différents obtenus par Merrill [7] sont sans doute dus au détecteur à ouverture relativement large $\left(2^{\circ}\right)$ utilisé qui ne permettait pas, comme ici, de résoudre dans l'espace contributions élastiques et inélastiques.

Ayant ainsi isolé la composante élastique de la diffusion, il faut encore nous assurer de sa cohérence. L'absence de pic de diffraction d'ordre supérieur à 00 n'est pas, à notre avis, un argument décisif contre cette cohérence car elle peut sans doute s'expliquer par la faible amplitude de la composante périodique du potentiel le long de la surface pour les métaux [9]. Une meilleure preuve consiste à vérifier si l'intensité $(I)$ du pic spéculaire est bien proportionnelle au facteur de Debye-Waller :

$$
\operatorname{Ln}\left(I / I_{0}\right)=-2 W
$$

$I_{0}$ étant l'intensité du faisceau incident. (Dans la limite classique l'intensité du pic spéculaire extrapolée à $0 \mathrm{~K}$ sera égale à $I_{0}$ puisqu'il n'y a pas de pic d'ordre supérieur.)

On sait que :

$$
2 W=\left\langle\left(\mathbf{u}_{\mathrm{s}} \cdot \Delta \mathbf{k}\right)^{2}\right\rangle
$$

avec $\mathbf{u}_{\mathrm{s}}$ déplacement d'un atome de surface,

$\Delta \mathbf{k}$ variation de vecteur d'onde de l'atome diffusé soit pour le pic 00

$$
2 W=\left\langle u_{\mathrm{s}_{\perp}}^{2}\right\rangle \cdot\left(\Delta k_{\perp}\right)^{2}
$$

avec $\left\langle u_{\mathrm{s}_{\downarrow}}^{2}\right\rangle$ déplacement quadratique moyen normal de l'atome de surface,

$\Delta k_{\perp} \quad$ variation de la composante normale du vecteur d'onde de l'atome diffusé.

$\left\langle u_{\mathrm{s}_{\perp}}^{2}\right\rangle$ s'exprime au moyen de $\Theta$ d'après (1) et

$$
\left(\Delta k_{\perp}\right)^{2}=\frac{8 m_{\mathrm{G}} E_{\mathrm{G}}}{\hbar^{2}}\left(\cos ^{2} \theta_{\mathrm{i}}+\frac{D}{E_{\mathrm{G}}}\right)
$$

$m_{\mathbf{G}}:$ masse de l'atome incident.

Le terme $D / E_{\mathrm{G}}$ tient compte de l'influence du puits de potentiel (profondeur $D$ ). On considère depuis les travaux de Beeby [10] que la partie attractive du potentiel dont la portée est relativement longue reste stationnaire et n'a d'autre effet que de modifier la vitesse des atomes au voisinage de la surface. La diffusion proprement dite s'effectue sur la partie répulsive de ce potentiel. En combinant (1), (2), (3) et (4) il vient :

$$
\operatorname{Ln}\left(I / I_{0}\right)=-48 \frac{m_{\mathrm{G}}}{m_{\mathrm{s}}} \frac{E_{\mathrm{G}}}{2 k \Theta^{2}} T_{\mathrm{S}}\left(\cos ^{2} \theta_{\mathrm{i}}+D / E_{\mathrm{G}}\right) .
$$


Sur la figure $2 a$ nous avons porté

$$
\operatorname{Ln}\left(I / I_{0}\right) /\left(\cos ^{2} \theta_{\mathrm{i}}+D / E_{\mathrm{G}}\right)
$$

en fonction de $T_{\mathrm{S}}$ pour $E_{\mathrm{G}}=0,063 \mathrm{eV}$ et $\theta_{\mathrm{i}}$ variant de 10 à $60^{\circ}$. La valeur de $D$ a été optimisée pour que tous les points tombent sur une même courbe. Ceci conduit à $D=0,009 \pm 0,002 \mathrm{eV}$. Cette valeur est tout à fait raisonnable pour une interaction héliumcuivre. Entre 373 et $650 \mathrm{~K}$ cette courbe est une droite passant par 0 pour $T=0 \mathrm{~K}$. Ceci justifie le modèle adopté (diffusion élastique cohérente). On montre en outre que la surface participe en totalité à cette diffusion cohérente, ce qui est une preuve supplémentaire de sa propreté. La courbure qui apparaît vers $700 \mathrm{~K}$ et au-dessus peut être attribuée aux effets d'anharmonicité en bon accord avec les mesures de volume (diffraction de rayons $\mathrm{X}$ [11]) ou de surface (D.E.L. [2]).

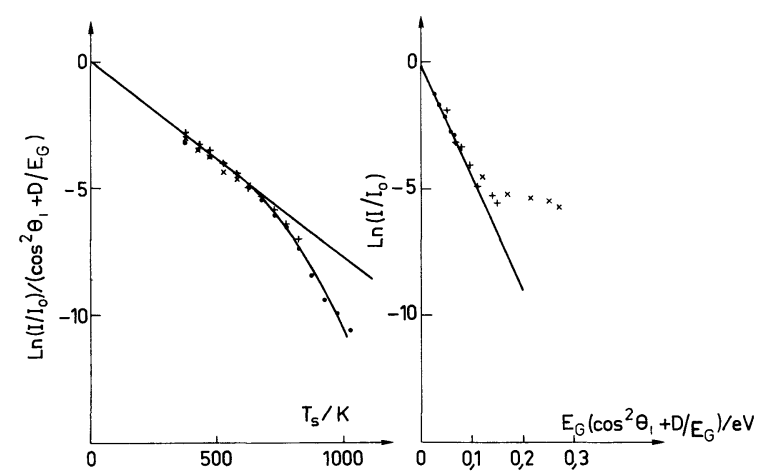

a)

b)

FIG. 2. $-a) \operatorname{Ln}\left(I / I_{0}\right) /\left(\cos ^{2} \theta_{\mathrm{i}}+D / E_{\mathrm{G}}\right)=f\left(T_{\mathrm{S}}\right)$

$$
\begin{aligned}
E_{\mathrm{G}}=0,063 \mathrm{eV} \quad \theta_{\mathrm{i}}= & 60^{\circ} \bullet \\
& 40^{\circ}+ \\
& 10^{\circ} \times
\end{aligned}
$$

b) $\operatorname{Ln}\left(I / I_{0}\right)=f\left(E_{\mathrm{G}}\left[\cos ^{2} \theta_{\mathrm{i}}+D / E_{\mathrm{G}}\right]\right)$

$$
\begin{aligned}
T_{\mathrm{S}}=373 \mathrm{~K} \quad E_{\mathrm{G}}= & 0,063 \mathrm{eV} \bullet \\
& 0,154 \mathrm{eV}+ \\
& 0,270 \mathrm{eV} \times .
\end{aligned}
$$

On a également étudié l'influence de l'énergie incidente $E_{\mathrm{G}}$ en portant sur la figure $2 b \operatorname{Ln}\left(I / I_{0}\right)$ en fonction de $E_{\mathrm{G}}\left(\cos ^{2} \theta_{\mathrm{i}}+D / E_{\mathrm{G}}\right)$ pour $T=373 \mathrm{~K}, \theta_{\mathrm{i}}$ variant de 10 à $60^{\circ}$ et $E_{\mathrm{G}}=0,063 ; 0,154 ; 0,27 \mathrm{eV}$.

Les points s'alignent bien sur une droite passant par l'origine sauf pour les énergies élevées et les faibles incidences où l'écart observé correspond aux effets inélastiques signalés plus haut.

De la pente de l'une ou l'autre des courbes $(2 a$ ou $2 b$ ) on tire une température de Debye apparente $\Theta_{\text {app }}=370 \pm 10 \mathrm{~K}$. Cette valeur ne correspond pas à la température de Debye de surface $\Theta_{\mathrm{S}_{\perp}}$ à laquelle on doit s'attendre. Il faut ici rappeler que le diamètre de l'atome d'hélium $(2,55 \AA)$ est comparable à la maille du cristal (2,56 $\AA$ ). Dans ces conditions l'atome d'hélium doit être simultanément en interaction avec plusieurs atomes de surface. Ainsi le centre diffuseur ne sera-t-il pas un atome isolé de la surface mais une cellule en comportant plusieurs, $\Theta_{\text {app }}$ reflétera alors les déplacements moyens de l'ensemble de cette cellule. Cette influence d'un facteur géométrique avait déjà été signalée par Weinberg [16] pour expliquer l'absence de diffraction de l'hélium sur les métaux.

Hoinkes, Nahr et Wilsch [12] ont rencontré le même problème dans la diffraction de l'hydrogène atomique par le fluorure de lithium. Ils ont proposé un modèle dans lequel ils introduisent des collisions avec 1, 2, 3 ou 4 atomes suivant les valeurs du paramètre d'impact, chaque atome de surface étant considéré comme un oscillateur harmonique indépendant ce qui, dans notre cas, conduit à $\Theta_{\mathrm{S}_{\perp}}=150 \mathrm{~K}$, valeur certainement trop faible. En fait ce modèle a l'inconvénient d'introduire les collisions à plusieurs atomes d'une manière assez artificielle et de négliger les corrélations entre les déplacements des atomes voisins. Pour lever ces difficultés, nous avons utilisé une méthode s'appuyant sur le concept de pseudo-potentiel utilisé avec succès pour l'interprétation de la diffraction des neutrons. Ici nous tenons compte de l'interaction de l'atome avec une cellule carrée $(\mathrm{L})$ comportant quatre atomes de surface (cellule de base du plan (100)) en introduisant le pseudo-potentiel

$$
V=\sum_{\mathrm{L}} v_{\mathrm{L}} \delta\left(\mathbf{r}_{0}-\left(\mathbf{r}_{1}+\mathbf{r}_{2}+\mathbf{r}_{3}+\mathbf{r}_{4}\right) / 4\right)
$$

où $v_{\mathrm{L}}$ est une constante, $\mathbf{r}_{0}$ est le vecteur position de l'atome incident et $\mathbf{r}_{1}, \mathbf{r}_{2}, \mathbf{r}_{3}, \mathbf{r}_{4}$ les vecteurs position des atomes de la cellule. La sommation s'étend à toutes les cellules de la surface. Le calcul dans l'approximation de Born se déroule exactement comme pour les neutrons [13] et conduit à un facteur de Debye-Waller de la forme :

$$
2 W=\left\langle u_{\mathrm{L}}^{2}\right\rangle \cdot \Delta k_{\perp}^{2}
$$

avec

$$
\left\langle u_{\mathrm{L}}^{2}\right\rangle=\frac{1}{4}\left\langle\left(u_{1}^{z}\right)^{2}\right\rangle+\frac{1}{2}\left\langle u_{1}^{z} u_{2}^{z}\right\rangle+\frac{1}{4}\left\langle u_{1}^{z} u_{3}^{z}\right\rangle
$$

$u_{1}^{z}, u_{2}^{z}, u_{3}^{z}$ représentant respectivement les composantes normales du déplacement d'un atome de surface, de son premier voisin et de son second voisin.

Les calculs de $\left\langle\left(u_{1}^{z}\right)^{2}\right\rangle,\left\langle u_{1}^{z} u_{2}^{z}\right\rangle,\left\langle u_{1}^{z} u_{3}^{z}\right\rangle$ ont été effectués par G. Armand [14] à l'aide des coefficients générateurs de la fonction de Green d'un cristal cubique à faces centrées semi-infini ne comportant que des forces centrales harmoniques entre plus proches voisins.

Si l'on ajuste la constante de force pour retrouver la température de Debye du volume $\left(\Theta_{\mathrm{V}}=330 \mathrm{~K}\right)$ le calcul donne :

$$
\Theta_{\mathrm{app}}=333 \mathrm{~K} \text { et } \Theta_{\mathrm{S}_{\perp}}=203 \mathrm{~K} \text {. }
$$

Malgré sa simplicité ce modèle fournit donc un bon ordre de grandeur à la fois pour la température de Debye de surface et la température apparente déduite du facteur de Debye-Waller expérimental. 
Il montre que l'hypothèse d'une interaction héliumcuivre (100) faisant intervenir quatre atomes de surface est très vraisemblable.

Remerciements. - Nous tenons à remercier ici MM. Degras et Manus de l'intérêt qu'ils ont porté à ce travail, MM. Beullard, Le Cruër, Lefort pour leur contribution à la mise au point et à la conduite de l'expérience et tout particulièrement $M$. Domange de l'Ecole Supérieure de Chimie pour s'être chargé de la désulfuration des échantillons de cuivre utilisés ainsi que M. Campargue et son équipe du Service de Chimie Physique à Saclay qui a étudié et réalisé le jet à énergie variable.

\section{Bibliographie}

[1] Masri, P., Dobrzynski, L., Surf. Sci. 32 (1972) 623.

[2] REID, R. J., Surf. Sci. 29 (1972) 623.

[3] Weinberg, W. H., MerRill, R. P., Adsorption-Desorption Phenomena, F. Ricca Editeur (Academic Press, New York) 1972 , p. 151

[4] Weinberg, W. H., J. Chem. Phys. 57 (1972) 5463.

[5] Lapujoulade, J., Lejay, Y., J. Chem. Phys. 63 (1975) 1389.

[6] Comsa, G., J. Phys. C: Solid St. Phys. 6 (1973) 2648.

[7] Stoll, A. G., Smith, D. L., Merrill, R. P., J. Chem. Phys. 54 (1971) 163.

[8] Campargue, R., Lebehot, A., Lemonnier, J. C., Marette, D., Pebay, J., 5th Symp. Int. sur les Jets Moléculaires, Nice (1975).
[9] Garibaldi, U., Levi, A. C., Spadacini, R., Tommei, G. E., Surf. Sci. 48 (1975) 649.

10] Beeby, J. L., J. Phys. C : Solid St. Phys. 4 (1971) 359.

[11] Owen, E. A., Williams, R. W., Proc. R. Soc. A 188 (1947) 509.

[12] Hoinkes, H., Nahr, H., WilsCh, H., Surf. Sci. 33 (1972) 516.

[13] Maradudin, A. A., Montroll, E. W., Weiss, G. H., Ipatova, I. P., Theory of lattice dynamics in the harmonic approximation (Academic Press, New York), 1971, p. 325.

[14] Armand, G., J. Physique 36 (1975) 75.

[15] Weinderg, W. H., Adv. Colloid. Interface Sci. 4 (1975) 301.

[16] Weinberg, W. H., J. Phys. C : Solid St. Phys. 5 (1972) 2098. 